\title{
Carbon Dioxide-Mediated Generation of Hybrid Nanoparticles for Improved Bioavailability of Protein Kinase Inhibitors
}

\author{
Gérald Jesson • Magnus Brisander • Per Andersson • Mustafa Demirbüker • Helene Derand • Hans Lennernäs • Martin Malmsten
}

Received: 31 May 2013 / Accepted: 9 August 2013 / Published online: 30 August 2013

(C) Springer Science+Business Media New York 2013

\begin{abstract}
Purpose A versatile methodology is demonstrated for improving dissolution kinetics, gastrointestinal (GI) absorption, and bioavailability of protein kinase inhibitors (PKIs).

Methods The approach is based on nanoparticle precipitation by sub- or supercritical $\mathrm{CO}_{2}$ together with a matrix-forming polymer, incorporating surfactants either during or after nanoparticle formation. Notably, striking synergistic effects between hybrid PKI/ polymer nanoparticles and surfactant added after particle formation is investigated.

Results The hybrid nanoparticles, consisting of amorphous PKI embedded in a polymer matrix (also after 12 months), display dramatically increased release rate of nilotinib in both simulated gastric fluid and simulated intestinal fluid, particularly when surfactants are present on the hybrid nanoparticle surface. Similar results indicated flexibility of the approach regarding polymer identity, drug load, and choice of surfactant. The translation of the increased dissolution rate found in vitro into improved Gl absorption and bioavalilability in vivo was demonstrated for male beagle dogs, where a $730 \%$ increase in the $\mathrm{AUC}_{0-24 \mathrm{~h}}$ was observed compared to the benchmark formulation. Finally, the generality of the formulation approach taken was demonstrated for a range of PKIs.
\end{abstract}

Conclusions Hybrid nanoparticles combined with surfactant represent a promising approach for improving PKI dissolution rate, providing increased $\mathrm{Gl}$ absorption and bioavailability following oral administration.

\footnotetext{
Electronic supplementary material The online version of this article (doi: | 0. I007/s | |095-0 | 3- | | 9| -4) contains supplementary material, which is available to authorized users.
}

G. Jesson • M. Brisander $\cdot$ P. Andersson • M. Demirbüker $\cdot H$. Derand

XSpray Microparticles AB, Fogdevreten 2B, I7I 65 Solna, Sweden

H. Lennernäs • M. Malmsten ( $\varangle)$

Department of Pharmacy, Uppsala University, 75I23 Uppsala, Sweden

e-mail: martin.malmsten@farmaci.uu.se
KEY WORDS bioavailability · carbon dioxide $\cdot$ hybrid . nanoparticle · protein kinase inhibitor

\section{INTRODUCTION}

Dysregulation of protein kinases by mutation, gene rearrangement, amplification, and overexpression has been implicated in the development and progression of human cancers (1). Protein kinases are frequently grouped according to the amino acid, which phosphorylation they inhibit. Most kinases act on both serine and threonine, while tyrosine kinases act on tyrosine, and a number (dual-specificity kinases) on all three. There are also protein kinases, which phosphorylate other amino acids, including histidine. For indications caused by the overexpression or up-regulation of protein kinases, protein kinase inhibitors (PKIs) have attracted considerable interest as therapeutics. For example, tyrosine kinase inhibitors have been shown to be effective anti-tumor and anti-leukemic agents, even if the gastrointestinal absorption is low and highly variable with present formulations (2). Thus, PKIs are generally weak bases, which dissolve only slightly at low $\mathrm{pH}$ (typically $100-1,000 \mathrm{mg} / \mathrm{L}$ ), and are practically insoluble at neutral $\mathrm{pH}$ (typically $0.1-10 \mathrm{mg} / \mathrm{L}$ ). As a result of this, PKIs display poor bioavailability following oral administration. Consequently, enhancing the solubility and/or dissolution rate of PKIs is key for improving the bioavailability and efficacy/safety ratio of this class of anticancer drugs.

Several methods for improving the dissolution characteristics of poorly water-soluble drugs have been reported, including micronization, as well as the formation of solvates, salts, complexes, and nanoparticles. Nanoparticular formulations provide opportunities of increased dissolution rate of sparingly soluble drugs through decreased particle size (and correspondingly higher Laplace pressure), and through destabilization of the crystalline state. Drug-containing nanoparticles can be generated through a range of approaches, including top- 
down methodologies such as media milling and homogenization, as well as bottom-up approaches such as controlled nucleation and nanoparticle growth in confinement. Due to the high energy state of such nanoparticles, however, maintaining their state of dispersion requires stabilization, which can be achieved, e.g., either by adsorbing a stabilizing surfactant or polymer layer on the nanoparticle surface, or by embedding the nanoparticles in a matrix, thus being equivalent to solid dispersions in the microscale (3-5).

Hybrid nano- and microparticles may be prepared by various techniques, including spray-drying, spray-cooling, spray freeze-drying and electrospraying, all having their strengths and weaknesses. Alternatively, nanoparticle formation can be achieved by approaches involving sub- and supercritical $\mathrm{CO}_{2}$, which offer opportunities related to, e.g., efficient solvent removal, fast processing, and process scalability. In the particular case of PKIs, $\mathrm{CO}_{2}$ precipitation is a powerful technique since it can be combined with poorly volatile solvents, notably DMSO, which are among very few solvent able dissolve sufficient PKI concentrations to generate high drug loads in the resulting nanoparticles.

Some of these approaches are based on $\mathrm{CO}_{2}$ as a solvent, generating particles on expanding the system, while others are based on $\mathrm{CO}_{2}$ as an anti-solvent, in combination with one or several organic solvents (6-14). The former offer the best option in the idealized case, since supersaturation of the order of $10^{5}-10^{6}$ can be achieved at a time scale of $10^{-6}-10^{-4} \mathrm{~s}$, facilitating solvent-free generation of very small particles of a high degree of homogeneity. Due to the poor solvency displayed by most pharmaceutically interesting excipients (notably polymers and surfactants) in $\mathrm{CO}_{2}$, however, substantially larger flexibility is obtained when $\mathrm{CO}_{2}$ is used as an antisolvent rather than as a solvent (14-16). Examples of polymers used in the latter context include, e.g., polyvinyl alcohol, hydroxypropyl methylcellulose (HPMC), ethyl cellulose/ methyl cellulose, and polyesters, while solvents used include, e.g., DMSO, DMF, methylene chloride, and ethyl acetate $(14,16,17)$.

Even for such a seemingly simple system and process, however, the materials generated depend on a range of composition and processing conditions. For example, faster flow of $\mathrm{CO}_{2}$ has been reported to be favourable for reducing particle size as well as the residual solvent level in the case of DMSO (12). Furthermore, a range of composition parameters have been found to play a key role in determining the structure and function of polymer-based (nano)particles, including polymer crystallinity (18), glass transition temperature (19), and surface activity (20,21). Also, the drug load in such hybrid nanoparticles critically affects their physical state, notably the crystallinity and crystal habit.

Given the need for improved gastrointestinal (GI) absorption and bioavailability of PKIs, as well as the opportunities offered by sub- and supercritical $\mathrm{CO}_{2}$ as anti-solvent, we here report a versatile formulation strategy based on hybrid nanoparticles formed by PKIs in the presence of matrixforming polymers and swelling/solubilizing surfactants. In doing so, we investigate effects of polymer concentration and drug load, as well as of surfactant presence and processing conditions. Notably, the manuscript reports on striking synergistic effects between hybrid PKI/polymer nanoparticles and surfactant added after particle formation. For selected formulations, detailed physicochemical characterization is provided through X-ray diffraction, modulated differential scanning calorimetry, vapor sorption measurements, and electron microscopy, and increased bioavailability demonstrated as proof of concept in male beagle dogs. Finally, generalization of the approach to other PKIs is demonstrated.

\section{MATERIALS AND METHODS}

\section{Chemicals}

Hydroxypropyl methylcellulose phtalate (HPMCP; HP55) was from Shin-Etsu (Tokyo, Japan), while polyvinyl acetate phthalate (PVAP; 2138 Clear) was from Colorcon (Harleysville, USA), polyvinylpyrollidone (PVP; K90) from Sigma-Aldrich (St. Louis, USA), 2-hydroxypropyl- $\beta$-cyclodextrin from Alfa Aesar (Karlsruhe, Germany), methacrylic acidmethyl methacrylate copolymer (1:1) (Eudragit L100) from Evonik Industries (Darmstadt, Germany), poly(ethylene oxide)-poly(propylene oxide)-poly(ethylene oxide) block copolymer (Lutrol F127) from BASF (Ludwigshafen, Germany), hydroxypropyl methylcellulose USP2910 (Pharmacoat 645) from Shin-Etsu Chemical Co. (Tokyo, Japan), hydroxypropyl methylcellulose USP2910 (Methocel E15) from Dow Chemical Company (Midland, USA), and hydroxypropyl methylcellulose acetate succinate (HPMC AS) from Shin-Etsu Chemical Co. (Tokyo, Japan). Polyvinylcaprolactonepolyvinylacetate-polyethylene glycol (Soluplus) and D- $\alpha$ tocopherol polyethylene glycol 1000 succinate (TPGS) was from BASF (Ludwigshafen, Germany) and Sigma (St. Louis, USA), respectively. While Soluplus is strictly a block copolymer, it will here be referred to as "surfactant" for simplicity. Nilotinib base and $\mathrm{HCl}$ salt was from Hwasun Biotechnology Co. (Shanghai, China), while lapatinib, pazopanib, erlotinib, gefitinib, sorafenib, dasatinib, and sunitinib were all obtained from Tecoland (New Irvine, USA). Nilotinib hydrochloride monohydrate (Tasigna) was from Novartis (Basel, Switzerland) (lot 092014 S0028).

\section{SCF Apparatus}

Figure S1 shows a simplified representation of the supercritical fluid (SCF) processing system used for hybrid nanoparticle generation. The system consists of one pumping set-up for the 
solution containing the active ingredient (PKI dissolved in a solvent, together with excipients where applicable) and one for the anti-solvent $\left(\mathrm{CO}_{2}\right)$. The two set-ups are connected at a nozzle (located in the reactor) where particles are produced by anti-solvent precipitation. Particles are retained in the reactor by a filtering set-up. A back-pressure regulator is used after the reactor for pressure control. Each pumping set-up is equipped with separate flow and pressure meters for good process control. Before nanoparticle generation, $\mathrm{CO}_{2}$ and solvent are pumped through the system (at $100 \mathrm{~g} / \mathrm{min}$ and $1 \mathrm{~mL} / \mathrm{min}$, respectively) until flow rates, pressure (125 bar), and temperature $\left(15^{\circ} \mathrm{C}\right)$ have reached steady state. The solvent is then replaced by solvent containing the PKI (as well as excipients where applicable), and nanoparticles produced in the reactor. After completion of pumping of the PKI-containing solution, $\mathrm{CO}_{2}$ is pumped through the reactor in order to extract residual solvent from the reactor and the powder. Finally, the reactor is depressurized, and the sample collected.

\section{Scanning Electron Microscopy (SEM)}

Environmental SEM experiments were performed using a FEI-Philips-XL 30 TMP-PW 6635/45 microscope (Eindhoven, Netherlands), operating at 20-22 kV. For sample preparation, a Balzers SCD 050 (Balzers, Liechtenstein) sputtering equipment was employed to render the samples electrically conductive, thereby preventing image distortion from electrical charge build-up, and achieving better secondary electron emission. In doing so, argon gas was introduced to the specimen chamber. After repeated chamber flushing with argon, a pressure of 0.05-0.1 mbar was established. High voltage (giving $40 \mathrm{~mA}$ ) was subsequently applied to create a field between the target (cathode) and the specimen table (anode), resulting in glow discharge and release of metal atoms from the gold target. By this treatment, the specimen surface, even a very fissured one, is coated with a homogeneous metal layer $(\approx 10 \mathrm{~nm})$ of sufficient electrical conductivity for SEM examination.

\section{Laser Diffraction}

Particle size distributions were obtained by laser diffraction, using a Mastersizer 2000 (Malvern, Worcestershire, UK). Due to van der Waals interactions, as well as humidity-induced swelling of the polymer matrix, agglomeration of primary particles is likely. In order to reduce such primary particle agglomeration, but also to limit dissolution in the dispersion media, light diffraction measurements were performed, after sonication, in Volasil 244 (VWR International, Lutterworth, U.K.), using a refractive index of 1.394. The cuvette was initially filled with Volasil only, after which nanoparticles were added under stirring (500 rpm) until a suitable light scattering intensity was obtained. For each sample, triplicate measurements were performed at room temperature, and particle size distributions calculated based on a refractive index and an absorption of the nanoparticles of 1.50 and 0.001 , respectively.

\section{Z-Potential Measurements}

Measurements of z-potential of hybrid nanoparticles were performed by dynamic light scattering at a scattering angle of $173^{\circ}$, using a Zetasizer Nano ZS (Malvern Instruments, Malvern, UK). Measurements were performed in duplicate at $25^{\circ} \mathrm{C}$. For nilotinib/HPMCP (40/60 wt/wt), z-potential was determined (in $1 \mathrm{mM}$ Tris, $\mathrm{pH} 7.4$ ) to $-29.4 \pm 0.4$ and -28.5 $\pm 1.3 \mathrm{mV}$ for surfactant added before and after nanoparticle formation, respectively. While interpretation of z-potential data for diffuse nanoparticles is precluded by hydrodynamic (and swelling-dependent) interactions, these results nevertheless show that, apart from steric stabilization due to the swelling polymer matrix and the block copolymer surfactants, electrostatics contribute to the colloidal stabilization of these systems.

\section{X-Ray Powder Diffraction (XRD)}

XRD experiments were run on an X'Pert Pro diffractometer (PANanalytical, Almelo, Netherlands) set in BraggBrentano geometry. The diffractometer was equipped with $20 \mu \mathrm{m}$ nickel filter and an X'Celerator RTMS detector with an active length of $2.122^{\circ} 2 \theta$. A representative sample was placed on a zero background quartz single crystal specimen support (Siltronix, Archamps, France). Experiments were run using $\mathrm{Cu} \mathrm{K} \alpha$ radiation $(45 \mathrm{kV}$ and $40 \mathrm{~mA}$ ) at ambient temperature and humidity. Scans were run in continuous mode in the range $4.5-40^{\circ}$ (or 2-50 $2 \theta$ using automatic divergence and anti-scatter slits with observed length of $10 \mathrm{~mm}$, a common counting time of $299.72 \mathrm{~s}$, and step size of $0.0167^{\circ} 2 \theta$. Data collection was done using the application software X'Pert Data Collector V.2.2j and instrument control software V.2.1E, while pattern analysis was done using X'Pert Data Viewer V.1.2c (all software being from PANanalytical, Almelo, Netherlands).

\section{Dynamic Vapour Sorption (DVS)}

The hygroscopicity of the samples was studied by Dynamic Vapor Sorption Gravimetry (DVS), using a DVS-1 (Surface Measurement Systems, Alperton, UK). Approximately $10 \mathrm{mg}$ of the substance was weighed into a glass cup. The relative weight was recorded at $20 \mathrm{~s}$ interval when the target relative humidity (RH) over the sample was increased stepwise from $0 \%$ to $90 \%$, and then similarly decreased back to $0 \% \mathrm{RH}$, with $10 \%$ RH per step. Each sample was run in three 
consecutive full cycles. The condition to proceed to the next level of RH was a weight change below or equal to $0.002 \%$ within $15 \mathrm{~min}$, with a maximum total time per step of $24 \mathrm{~h}$. Due to slow equilibration in experiments of this type, the numbers obtained should be regarded as lower estimates of water uptake. The temperature was kept at $25^{\circ} \mathrm{C}$.

\section{Modulated Differential Scanning Calorimetry (mDSC)}

Modulated Differential Scanning Calorimetry (mDSC) analysis was run on a TA Instruments Model Q200 (New Castle, USA), equipped with a RC90 refrigerated cooling system (Home Automation, New Orleans, USA). Samples were weighed to $7 \pm 2 \mathrm{mg}$ in Tzero Low-mass aluminum pans and sealed with Tzero lids. They were then heated at a rate of $3^{\circ} \mathrm{C} / \mathrm{min}$ from 0 to $170^{\circ} \mathrm{C}$ with conventional modulation temperature amplitude of $1^{\circ} \mathrm{C}$ and a modulation period of $40 \mathrm{~s}$. Ultra-high purity nitrogen was used as purge gas at a flow rate of $50 \mathrm{~mL} / \mathrm{min}$. All data analyses were performed using TA Universal Analysis software, version 4.7A. Cell constant and temperature calibrations were conducted with the use of an indium standard prior to instrument operation. DSC results were evaluated in terms of both forward and reversing components of heat flow. As all notable thermal events were captured and more clearly illustrated by the reverse component of heat flow, the DSC results are presented as reversing heat flow.

\section{Determination of Surfactant Concentration}

Surfactant/block copolymer content was determined by HPLC-RI, using an Alliance 2695 equipped with a 2410 differential refractometer (Waters, Milford, USA). Chromatographic conditions used included an injection volume of $20 \mu \mathrm{l}$, and a mobile phase of dimethylformamide (DMF; Thermo Fisher Scientific, Waltham, USA) at $0.9 \mathrm{~mL} / \mathrm{min}$. The column used was a Pathfinder AS Silica $1003.5 \mu \mathrm{m}$ RP 4.6× 150 mm (Shimadzu Scientific Instruments, Columbia, USA), and chromatograms were evaluated using software from Clarity International (North Sydney, Australia). The amount of Soluplus in hybrid nanoparticles was determined by adding $0.100 \mathrm{~mL}$ sample solution $(1.07 \mathrm{mg} / \mathrm{mL}$ in DMF $)$ to six separate HPLC vials. To four vials, different amount $(0.050$, $0.100,0.200$, and $0.300 \mathrm{~mL}$ ) of Soluplus standard solution $(1.02 \mathrm{mg} / \mathrm{mL}$ in DMF) was added. DMF was then added to each vial to give a total volume of $1.00 \mathrm{~mL}$. The samples were run on above HPLC system. A standard curve was then constructed and the amount of Soluplus determined.

\section{Determination of Residual Solvent}

The amount of residual DMSO was determined by GG-MS, using a Varian 3800 GC, equipped with a Varian 1200 Single quadropole mass spectrometer (Varian, Paolo Alto, USA). A Zebron ZB 624 fused silica column $(60 \mathrm{~m} \times 0.25 \mathrm{~mm}$ id, $\mathrm{df}=$ $0.14 \mu \mathrm{m}$ ) (Phenomenex, Torrance, USA) was used, initially keeping the oven temperature at $70^{\circ} \mathrm{C}$ for $3 \mathrm{~min}$, followed by a temperature increase to $130^{\circ} \mathrm{C}$ at $3^{\circ} \mathrm{C} / \mathrm{min}$, then at $6^{\circ} \mathrm{C} /$ min to $200^{\circ} \mathrm{C}$, and finally to $250^{\circ} \mathrm{C}$ at $50^{\circ} \mathrm{C} / \mathrm{min}$, followed by a final hold. Helium was used as a carrier gas at $1.2 \mathrm{mM} / \mathrm{min}$, and samples were injected at $1 \mu \mathrm{L}$ at $275^{\circ} \mathrm{C}$ in a split flow $(100 \mathrm{~mL} / \mathrm{min})$ configuration. MS conditions included EI ionization, a scan range of $20-600 \mathrm{~m} / \mathrm{z}$, a filament current of $150 \mu \mathrm{A}$, an ion source temperature of $200^{\circ} \mathrm{C}$, and a manifold temperature of $40^{\circ} \mathrm{C}$. After construction of a standard curve, sample concentrations were determined.

\section{Dissolution (Batch)}

$3.5 \mathrm{mg}$ of nilotinib base equivalent was added to an $8 \mathrm{~mL}$ glass bottle, whereafter $7 \mathrm{~mL}$ of solution (fasted state simulated intestinal fluid (FaSSIF)) (22) was added. Bottles were then placed on a shaker (40 rpm) for dissolution. Samples of $500 \mu \mathrm{l}$ where taken after different times, and subsequently centrifuged at approximately $13,000 \mathrm{~g}$ for $3 \mathrm{~min}$. The resulting supernatant was then analyzed by HPLG (C18 column Eclipse, $4.6 \mathrm{~mm} \times 15 \mathrm{~cm}$, Agilent Technologies, Santa Clara, USA), with a mobile phase $(1 \mathrm{~mL} / \mathrm{min})$ consisting of methanol/acetonitrile/water/trifluoro acetic acid (23/23/ 44/0.1 (v/v)) (VWR/Prolabo, Leuven, Belgium), and detection at $254 \mathrm{~nm}$.

\section{Dissolution (USP4)}

Dissolution according to USP4 was performed by off line HPLC. The system used consisted of a Dissotest CE1 equipped with a cell for testing powder (Sotax, Allschwil, Switzerland), a Scantec 650 HPLC pump (Partille, Sweden), and an RM 20 LAUDA water bath (Artisan Technology Group, Champaign, USA). For dissolution in FaSSIF and SGF (Biorelevant, Croydon, U.K.) (22), an Agilent 1100 HPLC system was used, equipped with a degassing unit, a binary pump, a thermostated auto sampler, a column heater, and diode array detector, all from Agilent (Palo Alto, USA). An injection volume of $5 \mu \mathrm{L}$ was used, together with a mobile phase $(1.0 \mathrm{~mL} / \mathrm{min})$ of methanol/acetonitrile/water/trifluoro acetic acid $(23 / 23 / 44 / 0.1(\mathrm{v} / \mathrm{v}))$. The column used was a Zorbax Eclipse XDB-C18 $5 \mu \mathrm{m}$ 4,6 mm*150 mm (Agilent Technologies, Santa Clara, USA), and data was evaluated by Chemstation (Agilent, Palo Alto, USA). A sample containing $3.5 \mathrm{mg}$ PKI was placed in the dissolution cell. The cell was then placed in $37^{\circ} \mathrm{C}$ water bath. The pump was operated at $8 \mathrm{~mL} / \mathrm{min}$. Samples (approx $0.9 \mathrm{~mL}$ ) were collected at predetermined intervals, analyzed offline, and the amount determined through external calibration. 


\section{Animal Experiments}

Pharmacokinetic studies in beagle dogs were conducted under Animal Care and Use Committee (IACUC) protocols 11 IA6 and 12IA13. Samples ( $5 \mathrm{mg} / \mathrm{kg}$ ) for in vivo experiments were pre-filled size 0 hard gelatin capsules (Capsugel, Colmar, France) and stored in a desiccator at room temperature, protected from light, until use. Animal experiments were designed to focus on the performance of the best hybrid nanoparticle formulation in comparison with two control formulations, restricting studies of variations in drug load, polymer/surfactant concentration and type, as well as processing conditions, to in vitro investigations. As controls, a $0.2 \mathrm{mg} / \mathrm{mL}$ formulation consisting of $10 \%$ hydroxypropyl- $3-$ cyclodextrin in water (with $\mathrm{pH}$ and osmolarity adjustment) was used, as was a commercial benchmark formulation (Tasigna). The systemic exposure following oral administration was evaluated (non-blinded) in male beagle dogs. Each formulation was dosed in quadruplicate in each group for a total of 24 dogs (crossover). Animals were supplied with a commercial diet and water ad libitum prior to study initiation. Food was then withheld from the animals for a minimum of twelve hours before the study, as well as during the study until four hours post dose, when food was returned. For the hydroxypropyl- $\beta$-cyclodextrin formulation of nilotinib, animals received test compound by intravenous infusion for $30 \mathrm{~min}$. All other animals received a dose by capsule at time zero on the day of dosing. Five minutes prior to dosing, the $\mathrm{pH}$ of the stomach was neutralized using oral administration of $10 \mathrm{~mL}$ of a sodium bicarbonate solution in water $(100 \mathrm{mg} /$ $\mathrm{dog}, 10 \mathrm{mg} / \mathrm{mL}, 10 \mathrm{~mL} / \mathrm{dog}$ ). After dosing of the capsules, $50 \mathrm{~mL}$ of water was administered as a flush. Blood samples were collected via the jugular vein and placed into chilled glass microtainer tubes containing sodium heparin. Samples were centrifuged $\left(4^{\circ} \mathrm{C}\right)$ at $3,000 \mathrm{~g}$ for $5 \mathrm{~min}$. Plasma samples were then transferred into labeled polypropylene tubes, placed on dry ice, and stored in a freezer set to maintain $-60^{\circ} \mathrm{C}$ to $-80^{\circ} \mathrm{C}$. Nilotinib concentration was determined by LC-MS / MS using a single eight-point standard curve and quality control samples at three levels with six replicates each. Pharmacokinetic parameters were calculated from the time course of the plasma concentration. The maximum plasma concentration $\left(\mathrm{C}_{\max }\right)$ and time to the maximum plasma drug concentration $\left(t_{\max }\right)$ were calculated using the noncompartmental model, while the area under the plasma drug concentration-time curve from 0 to $24 \mathrm{~h}\left(\mathrm{AUC}_{0-24 \mathrm{~h}}\right)$ was calculated using the trapezoidal formula. The Mean Residence Time $\left(\mathrm{MRT}_{\text {last }}\right)$ was calculated from AUMC/AUC (AUMC being the area under the first moment curve) to the last observable time point $(24 \mathrm{~h})$, and the plasma half-life $\left(\mathrm{t}_{1 / 2}\right)$ from 0.693 slope of the terminal elimination phase. Data are reported as mean \pm standard deviation of means $(\mathrm{SD})$. A minimum of four animals per time point was used. All statistical tests were performed using Graphpad Prism (Version 4.00; Graphpad Software Inc., San Diego, CA). Student's t-test was performed at $95 \%$ confidence intervals, and a minimum $p$ value of 0.05 was used as the significance level.

\section{RESULTS}

Due to unique physicochemical properties, sub- and supercritical $\mathrm{CO}_{2}$ displays poor miscibility with a wide range of pharmaceutical excipients. While this limits the use of $\mathrm{CO}_{2}$ in nanoparticle formation based on expansion methodologies, this is a major advantage in nanoparticle formation through the use of $\mathrm{CO}_{2}$ as anti-solvent. Thus, efficient particle formation and solvent removal can be achieved also with solvents of high boiling point, e.g., DMSO, which are often last resort solvents for sparingly soluble drugs. Demonstrating the importance of the latter, Table $\mathrm{S} 1$ shows solubility estimates of a range of PKIs in a range of commonly used solvents. As can be seen, the PKIs investigated are characterized by limited to poor solubility in all solvents except DMSO. While DMSO is frequently used as solvent for a range of sparingly soluble drugs, it is also notoriously difficult to remove. With nanoparticle generation using $\mathrm{CO}_{2}$ as anti-solvent, however, even DMSO is efficiently removed. For example, residual DMSO in hybrid nanoparticles formed by nilotinib/ HPMCP (40/60 wt/wt) was found to be below $50 \mathrm{ppm}$ (results not shown). Such low levels of residual solvent are attractive from a safety perspective, but also from marginal solvent plastization, resulting in limited material re-organization after particle formation, and hence in an increased physical stability.

As demonstrated in Fig. 1, $\mathrm{CO}_{2}$-generated nilotinib particles are highly crystalline, with similar structural characteristics as unprocessed nilotinib, but with considerable smaller particle size. On co-precipitation in the presence of a polymer, drug incorporation in a polymer matrix is anticipated. Demonstrating this for nilotinib/ HPMCP particles (Fig. 2a, b), Fig. 2c shows XRD pattern at a (nilotinib/polymer) drug load of $40 \mathrm{wt} \%$. As can be seen, nilotinib is amorphous in the composite particles generated, and remains so even after at least 12 months of storage at $20^{\circ} \mathrm{C}$ (ongoing for multiple batches; for pazopanib and lapatinib the corresponding number is 12 and 14 months, respectively (ongoing)). Efficient drug encapsulation is obtained up to a drug-load of $\approx 60 \mathrm{wt} \%$, as inferred from XRD data on amorphicity/crystalinity (Fig. 2c) and from the drug-load-dependent dissolution rate (Fig. 3b). In line with this, modulated differential scanning calorimetry (Fig. 2d) displays a high single glass transition temperature at $127^{\circ} \mathrm{C}$, reporting on an amorphous phase of good stability. Quantitatively, the $\mathrm{T}_{\mathrm{g}}$ obtained for nilotinib/ $\operatorname{HPMCP}(40$ / $60)$ is similar to that of HPMCP in the absence of nilotinib. Further indicating that the continuous phase in the hybrid nanoparticles is indeed formed by the polymer, dynamic 
Fig. I Effect of $\mathrm{CO}_{2}$ processing on crystallinity and morphology of nilotinib. Shown in (a) and (b) are XRD and SEM data for unprocessed (left) and $\mathrm{CO}_{2}^{-}$ precipitated (right) nilotinib.
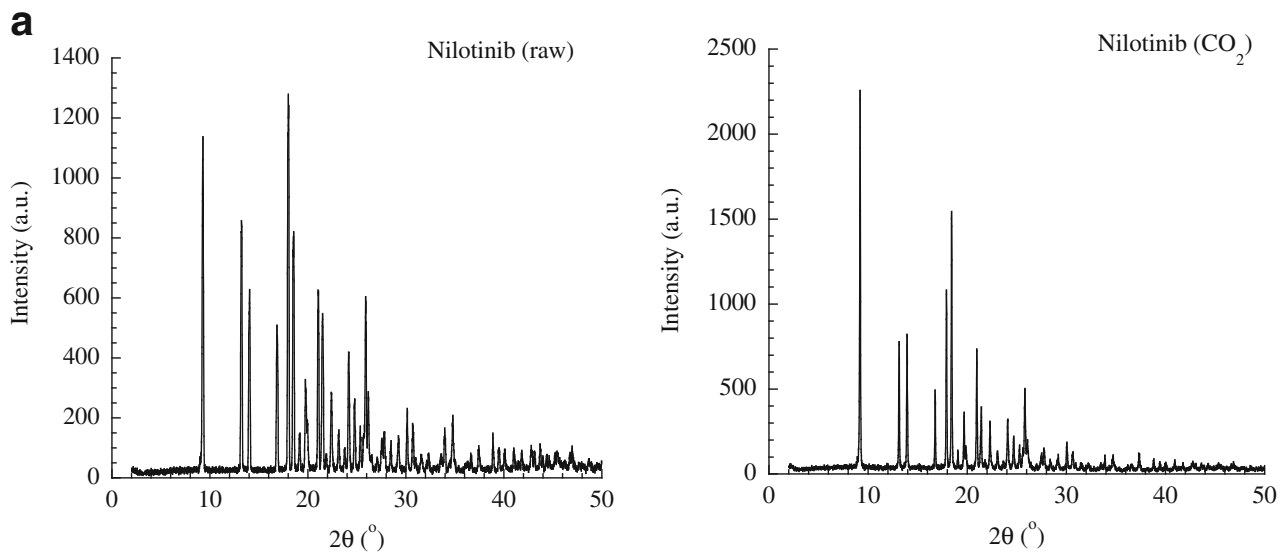

b

\section{$\mathrm{CO}_{2}$-precipitated}

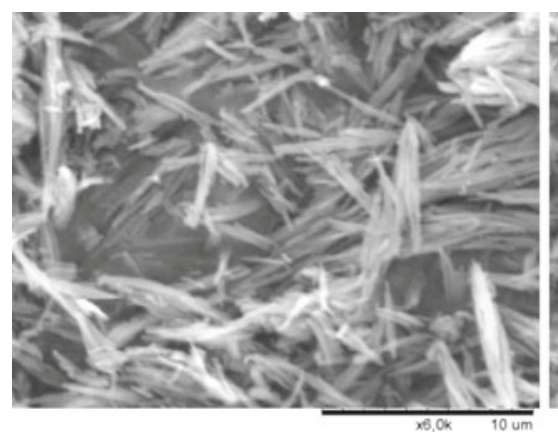

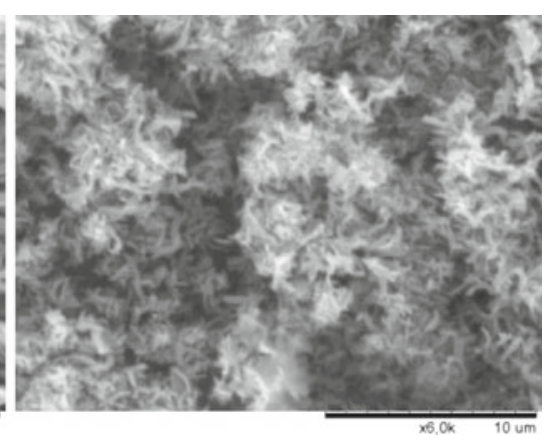

vapor sorption (Fig. 2e) shows a fair amount of water uptake with increasing relative humidity, but again no sign of humidity-induced phase transitions. (XRD analysis confirmed that the hybrid nanoparticles remained amorphous after these experiments (results not shown).) This is in stark contrast to nilotinib raw material, which displays much less water uptake. Together, the small variation in $\mathrm{T}_{\mathrm{g}}$, also at high drug load, the nanoparticle amorphicity, and a water uptake higher than that expected from the weighted average between the HPMCP and nilotinib components, suggest that nilotinib is present as small disordered domains within the hybrid nanoparticles, rather than being molecularly distributed $(23,24)$.

In order to get further indication on the performance of the hybrid nanoparticles, in vitro dissolution kinetics of nilotinib was monitored. As can be seen in Fig. 3a, nilotinib/ HPMCP nanoparticles display much higher dissolution rate than both nilotinib raw material and the physical mixture of nilotinib and HPMCP. These results are therefore compatible with the physicochemical characterization discussed above. It should here be noted that HPMCP is not the only matrix-forming polymer able to achieve dramatic improvement in nilotinib dissolution kinetics. Instead, similar results were obtained with PVAP and a number of other matrix-forming polymers
(Figure S2). The formulation approach also allows flexibility in polymer concentration/drug load, in favourable cases allowing drug loads up to $60 \%$ (Fig. 3b).

To further improve the dissolution kinetics from the hybrid nanoparticles, surfactant/block copolymer was added to the polymer/PKI formulation. Such surfactants/block copolymers not only solubilize the PKI through micelles formed $(25,26)$, but also cause swelling of the polymer matrix in aqueous solution, and promote dissolution of the outer layer once the latter is sufficiently swollen with water, thereby resulting in a reduced diffusion barrier and faster dissolution kinetics. As demonstrated in Figs. 4 and S3, respectively, Soluplus and TPGS both result in pronounced enhancement of the dissolution rate of nilotinib, compared to results obtained in the absence of surfactant. An important aspect of the presence of surfactant as dissolution enhancer in these systems, however, is that of how surfactant is introduced to the hybrid nanoparticle formulation. Thus, as shown in Figs. 4 and S3, surfactant dissolved in the organic solvent together with polymer and PKI prior to $\mathrm{CO}_{2}$-induced precipitation resulted in substantially smaller dissolution enhancement than surfactant added after PKI/polymer hybrid nanoparticle formation. Since chemical analysis showed that surfactant was present in high concentration also for the former particles (e.g., 
Fig. 2 Physicochemical characterization of nilotinib/ HPMCP hybrid nanoparticles (40/ $60 \mathrm{wt} / \mathrm{wt}$ ). Shown are results obtained by (a) laser diffraction, (b) SEM, (c) XRD, (d) modulated DSC, and (e) dynamic vapour sorption (RH: 0->90\%->0\%). In (d), water content was $2.1 \pm$ 0.1 wt $\%$ and $1.7 \pm 0.1$ wt $\%$ in the HPMC and the nilotinib/ HPMCP (40/60 wt/wt) sample, respectively. $\ln (\mathbf{c})$, retained amorphicity is demonstrated after 0 month and after 12 months of storage at $20^{\circ} \mathrm{C}$. Shown also in (c) is XRD data on (crystalline) nilotinib/ HPMCP at a 70 wt\% drug load. (XRD measurements after 12 months were obtained also at longer exposure times; Figure S4, Supporting Material.) a

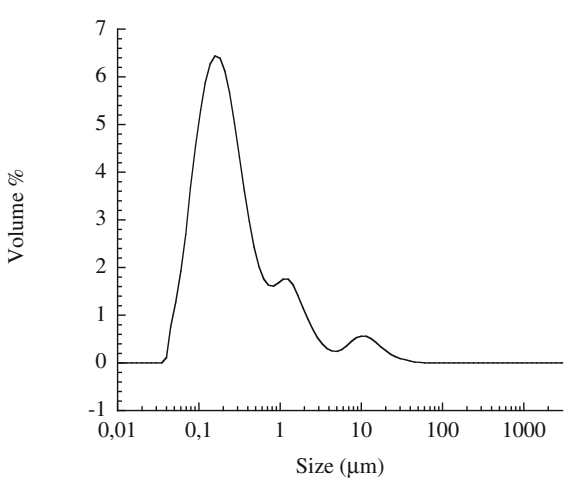

C

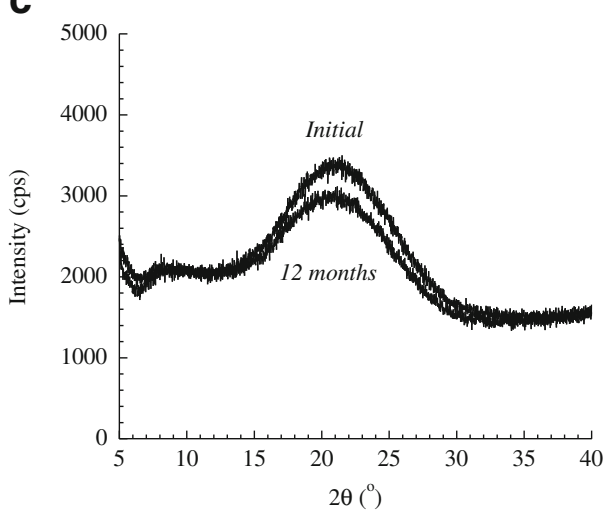

d

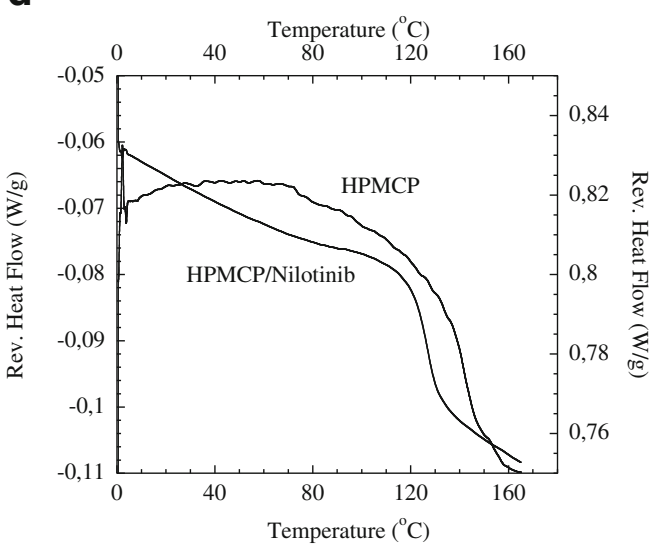

b

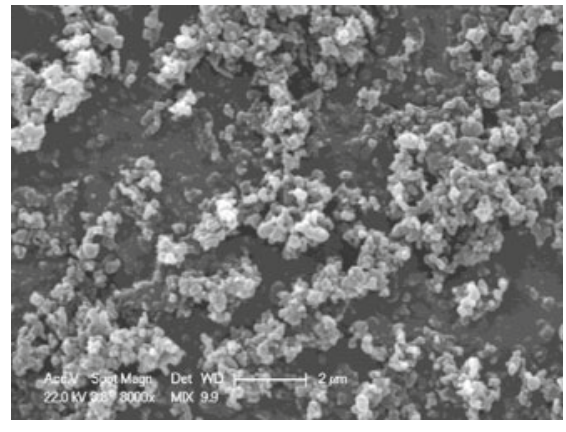

e

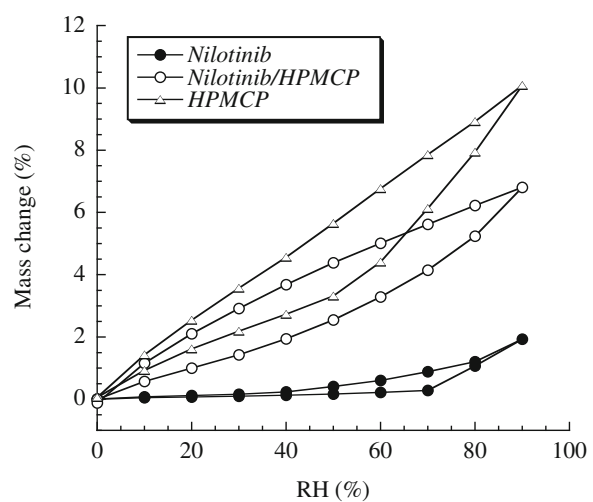

22 wt $\%$ in 25/38/36 nilotinib/ HPMCP /Soluplus), the origin of this effect is not only due to "material loss" at processing equipment surfaces or the like. Instead, the combined results indicate that surfactant added prior to particle formation is embedded in the hybrid nanoparticles, thus only partly accessible for maximum swelling and solubilization. In contrast, surfactant added after PKI/polymer particle formation is localized at the particle surface, thereby being optimally placed for promotion of both matrix swelling/dissolution and PKI solubilization. It is important to note, however, that the surfactant alone is not responsible for this effect, as the corresponding physical mixture between PKI, polymer, and surfactant does not result in high dissolution rate. Thus, despite having the same surfactant content, the physical mix of nilotinib, HPMCP (or PVAP), and Soluplus (or TPGS) displays $<10 \%$ dissolution compared to the hybrid nanoparticles (Figs. 4a and S3). Clearly, therefore, it is the combined effect of the matrix (resulting in small amorphous embedded PKI nanodomains in the polymer matrix) and the surfactant (causing matrix swelling/dissolution and enabling PKI solubilization in released micelles), which result in the strong enhancement of in vitro dissolution rate observed. 
a

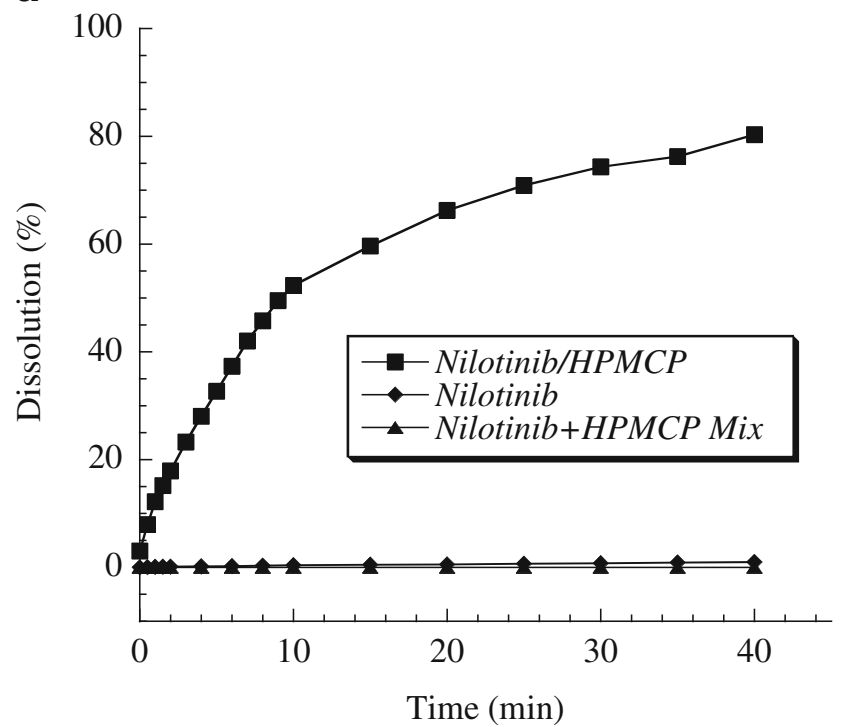

b

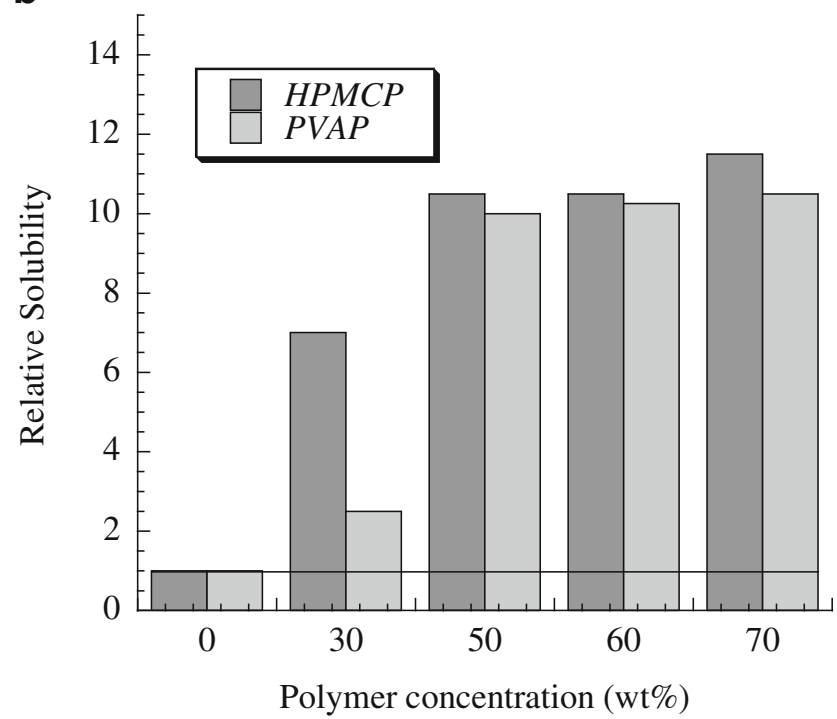

Fig. 3 (a) Role of polymer matrix for (USP4) dissolution kinetics of nilotinib in FaSSIF. Shown are dissolution curves for nilotinib/ HPMCP hybrid nanoparticles, as well as data for the corresponding physical mixture between nilotinib and HPMCP, both at a nilotinib/polymer ratio of 40/60 wt/wt, as well as data for nilotinib raw material. (b) Effect of polymer fraction of nilotinib/ polymer nanoparticles on dissolution rate in FaSSIF, expressed as "Relative Solubility", the ratio of the amount nilotinib dissolved from the hybrid nanoparticles after $90 \mathrm{~min}$ compared to that of raw nilotinib at a constant drug load of 40 wt\%.

As discussed above, the approach taken allows quite some flexibility in both polymer concentration and drug load, as well as in the nature of the polymer and that of the surfactant. As illustrated in Fig. 5, this flexibility holds also with regard to the drug itself, as a strong increase in dissolution kinetics is obtained for a range of PKIs. The relative enhancement in PKI dissolution kinetics, i.e., nilotinib $\approx$ lapatinib $>$ pazopanib $\approx$ erlotinib $>$ gefitinib $\approx$ sorafenib $>$ dasatinib $>$ sunitinib, is a

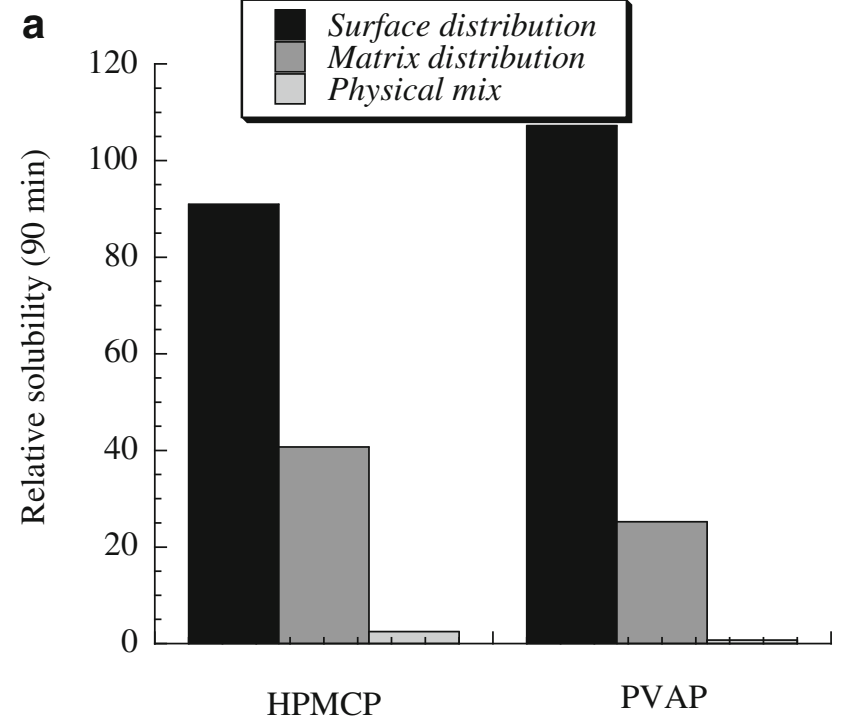

b

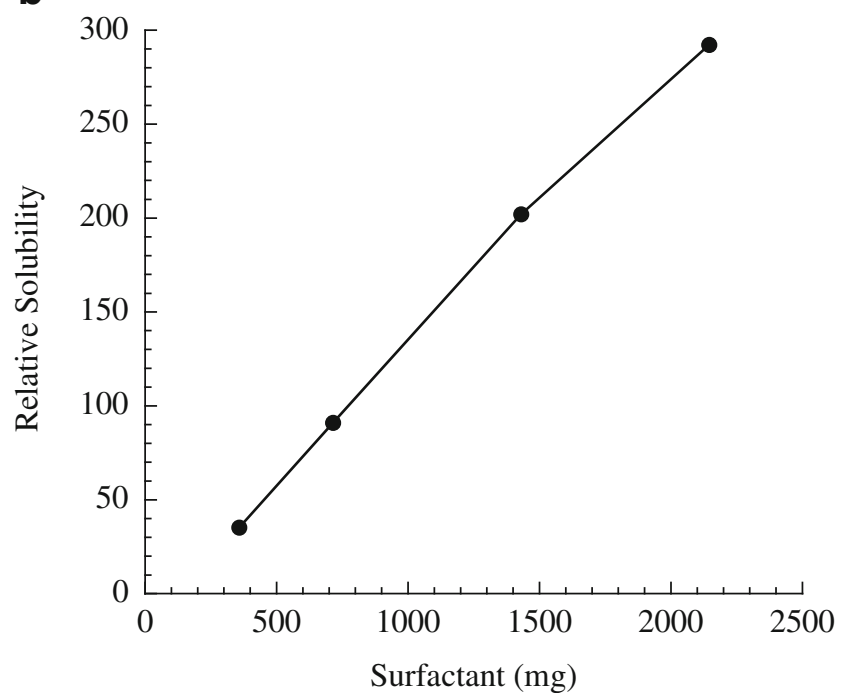

Fig. 4 Role of surfactant (Soluplus) on nilotinib (batch) dissolution kinetics in FaSSIF. The results are expressed as "Relative Solubility", the ratio of the amount nilotinib dissolved from the hybrid nanoparticles after 90 min compared to that of raw nilotinib at a constant drug load of $40 \mathrm{wt} \%$. Results are shown for surfactant added after nilotinib/polymer nanoparticle formation ("surface distribution"), for surfactant present together with polymer and nilotinib during nanoparticle formation ("matrix distribution"), and for the physical mix of raw nilotinib, polymer, and surfactant ("physical mix") (a). In (b), the effect of amount of surfactant added ("surface distribution") on the dissolution of nilotinib/ HPMCP (40/60 wt/wt) hybrid nanoparticles is shown as well.

reflection of both the properties of the nanoparticles and those of the PKI raw material. Although there is thus a spread in the extent of dissolution improvement reached with the different PKIs (the detailed analysis of which goes beyond the scope of the present investigation), the flexibility offered by the present formulation approach is clear.

In order to further investigate the relevance of the increased dissolution rate observed in vitro for in vivo GI 
a

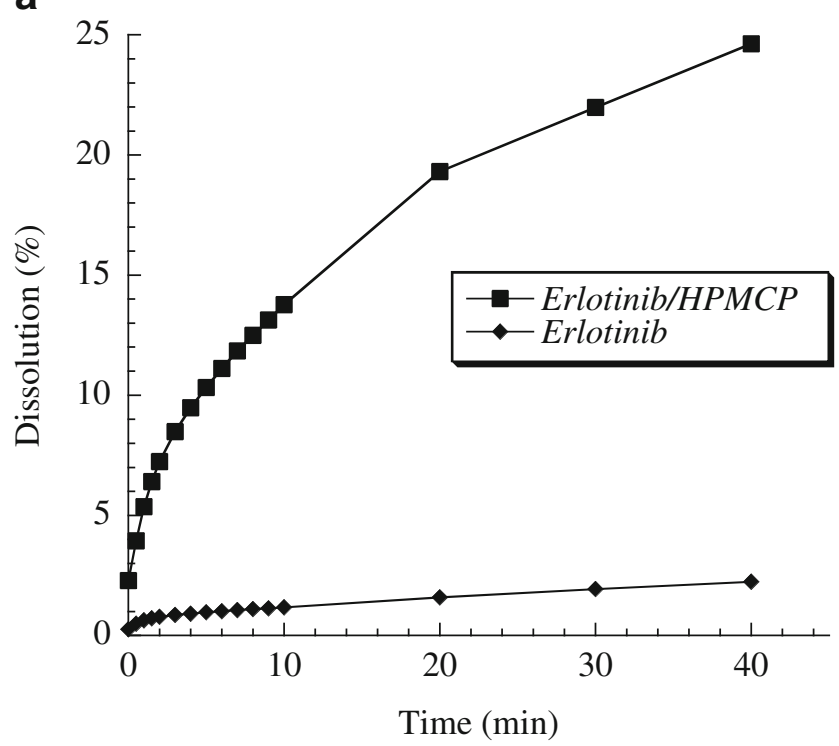

b

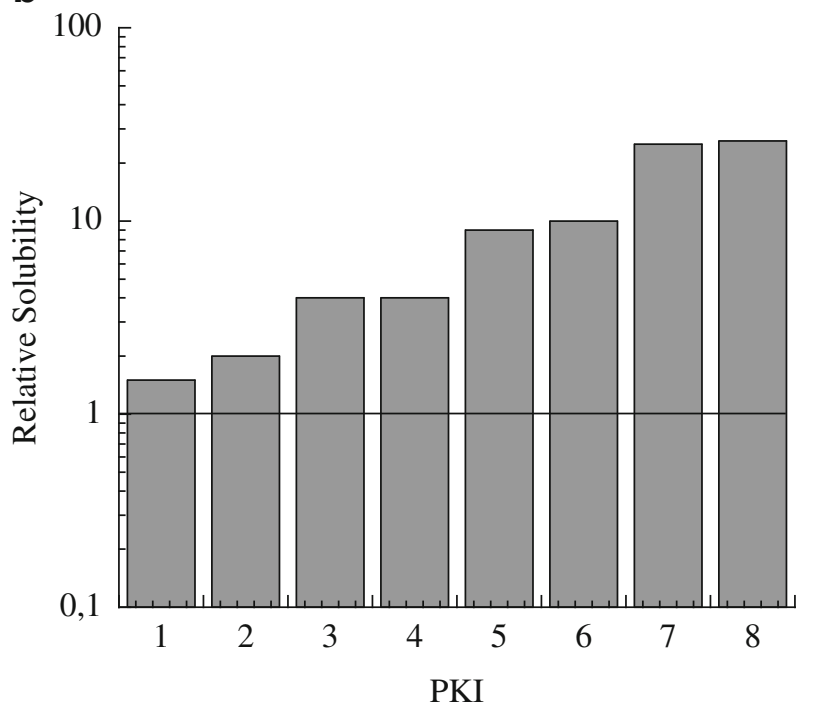

Fig. 5 Generalization of the formulation concept. Shown are dissolution data under sink conditions (USP4) for erlotinib/ HPMCP (35/65 wt/wt), compared to unprocessed erlotinib (a), as well as (b) the relative enhancement (compared to the unprocessed material) after $90 \mathrm{~min}$ in FaSSIF for a range of PKIs (I: sunitinib maleate; 2 : dasatinib; 3 : gefitinib; 4 : sorafenib tosylate; 5 : erlotinib $\mathrm{HCl}$; 6: pazopanib $\mathrm{HCl}$; 7 : nilotinib $\mathrm{HCl}$; 8: lapatinib ditosylate).

absorption and bioavailability following oral administration, the release of nilotinib was investigated for a selected formulation in simulated gastric fluid (SGF) and fasted state simulated intestinal fluid (FaSSIF). Generally being bases, PKIs display some solubility at low $\mathrm{pH}$ (e.g., in the stomach), but dramatically reduced solubility with increasing $\mathrm{pH}$. There is therefore a risk of re-precipitation of the PKI in the intestine, and it is thus of interest to investigate PKI dissolution rate under conditions simulating these latter conditions. As can be seen in Fig. 6, nilotinib indeed displays the expected higher dissolution rate in $\mathrm{SGF}$ due to its protonation at the low $\mathrm{pH}$ of SGF ( $\mathrm{pH} \approx 1.6$ ) (22). Importantly, however, dissolution rate remains good also in FaSSIF ( $\mathrm{pH} \approx 6.5$ ), in stark contrast to benchmark nilotinib formulation (Tasigna), which display very poor dissolution at the latter conditions.

After thus having demonstrated that nilotinib displays improved in vitro dissolution rate from the hybrid nanoparticle formulation in biorelevant media, compared to the clinically used benchmark formulation, the next step was to investigate whether the promising release results obtained in vitro translate also to in vivo GI absorption and bioavailability following oral administration. As shown in Fig. 7, the hybrid nanoparticle formulation of nilotinib increased GI absorption and bioavailability in male beagle dogs after oral administration of capsules containing the hybrid nanoparticles. Quantitatively, $\mathrm{AUC}_{0-24 \mathrm{~h}}$ for nilotinib/ HPMCP/Soluplus nanoparticles (26/34/45 wt/wt) was $730 \%$ of that of the benchmark nilotinib formulation (Tasigna). Furthermore, while the two formulations displayed comparable $t_{\max }$ and $\mathrm{MRT}_{\text {last }}$, the nanoparticle formulation displayed signtificantly higher $t_{1 / 2}$ (Table S2). In conclusion, therefore, the presently investigated approach is clearly of relevance also for enhancing bioavailability of PKIs following oral administration.

\section{DISCUSSION}

When forming polymer-drug particles by precipitation, particle structure and morphology depend on a number of parameters. While a more or less complete amorphization is most frequently observed (27), and found also in the presently

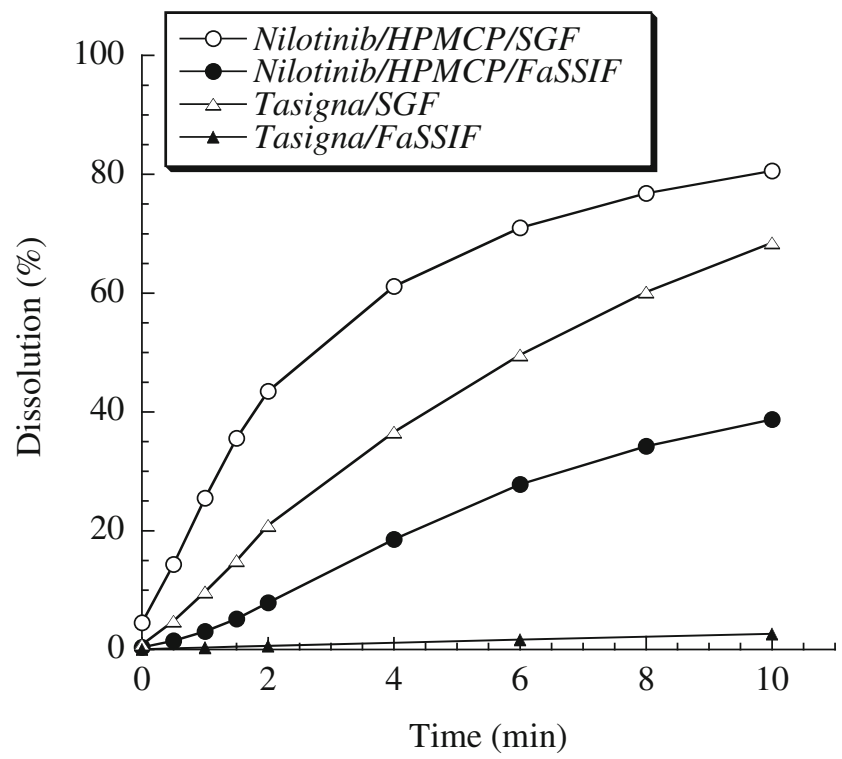

Fig. 6 Dissolution under sink conditions (USP4) of nilotinib/ HPMCP (40/60 $\mathrm{wt} / \mathrm{wt}$ ) in SGF and FaSSIF, compared to benchmark nilotinib (Tasigna). 
a

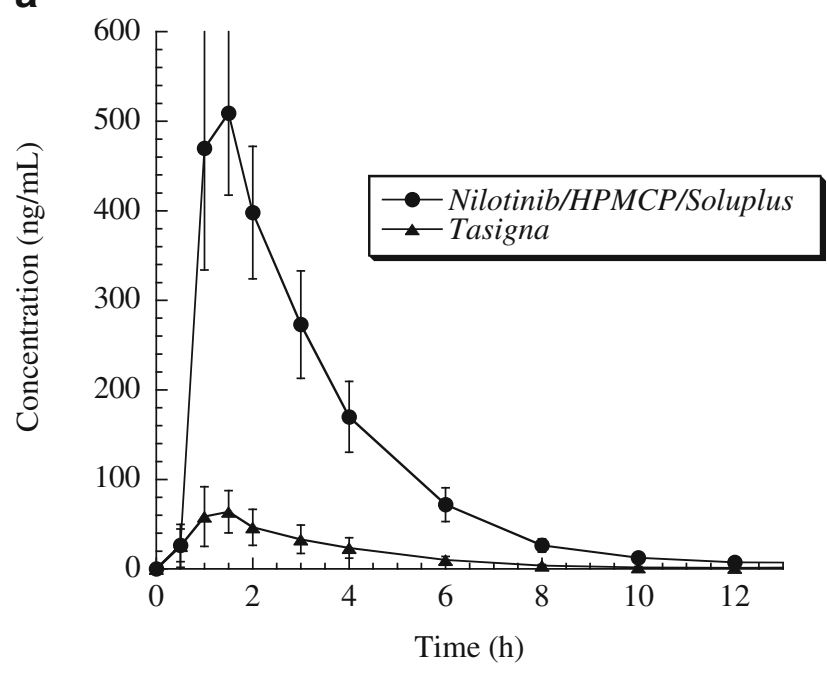

b

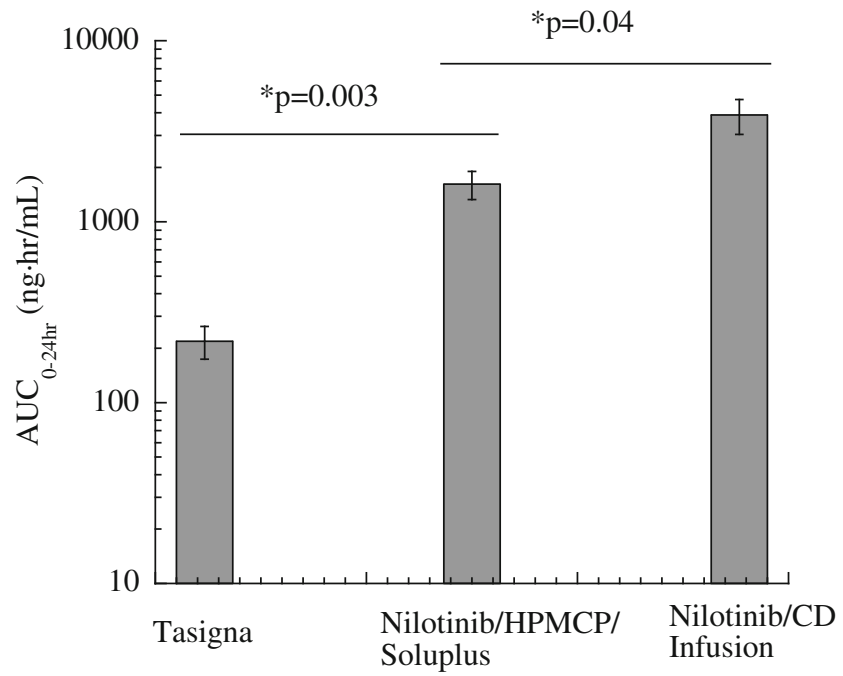

Fig. 7 Average plasma concentration versus time (a) and $\mathrm{AUC}_{0-24 \mathrm{~h}}(\mathbf{b})$ of nilotinib in male beagle dogs following oral administration of capsules containing either nilotinib/HPMCP/Soluplus (26/34/45 wt/wt) or benchmark nilotinib (Tasigna) at a dose of $5 \mathrm{mg} / \mathrm{kg}$. In (b), comparison with results obtained for nilotinib infused together with hydroxypropyl- $\beta$-cyclodextrin is included as well.

investigated systems (except at very high drug loads), also transitions from one ordered polymorph to another may be observed. As an example of the latter, Matsuda et al. observed a polymorphic transformation in phenylbuthasone, from the stable $\delta$ form to a metastable $\beta$ form (28). In the case of amorphization through nanoparticle formation, by use of $\mathrm{CO}_{2}$ or otherwise, it is frequently of interest to maintain this amorphous state, at least as long as a stable crystal form cannot be obtained at maintained particle size. In line with the excellent storage stability observed in the present investigation, Lim et al., previously demonstrated that coprecipitation of indomethacin with poly(vinylpyrrolidone) (PVP) provides stability against crystallization, the protective effect increasing with PVP molecular weight and concentration (27). Similarly, Kluge et al., investigated co-formulations between sparingly soluble phenytoin and PVP K30, using $\mathrm{CO}_{2}$ as anti-solvent, and found that particle crystallinity depended critically on the polymer/drug ratio (29). In line with the present finding of fully amorphous hybrid nanoparticles up to $50 \mathrm{wt} \%$, but crystallinity at $70 \%$ nilotinib, these authors found that phenytoin concentrations up to $40 \mathrm{wt} \%$ results in fully amorphous particles $(\approx 200-500 \mathrm{~nm})$, in analogy to particle formation of the polymer in the absence of drug. At higher drug concentrations, on the other hand, crystalline drug particles were obtained $(\approx 20-60 \mu \mathrm{m})$.

Essentially independent of production process, agglomeration and incomplete re-dispersion of dry nanoparticle powders frequently represents a formidable challenge. However, by coating the particle surfaces with a surfactant and/or a polymer, van der Waals and capillary interactions can be reduced, agglomeration suppressed, and re-dispersion facilitated (30). As suggested by the pronounced enhancement of PKI dissolution rate in the presence of surfactant, and more straightforwardly demonstrated by the light diffraction results, this is the case also for the presently investigated systems. As demonstrated, e.g. by Breitenbach et al., a high degree of polymer crystallinity may also reduce agglomeration due to reduced swelling (18). This was further demonstrated by Bodmeier et al., who found polymers with a low glass transition temperature to display pronounced agglomeration at room temperature, while agglomeration was less pronounced for polymers with a high glass transition temperature (19). These findings are therefore comparable with the present results, as the high glass transition demonstrated for the nilotinib/HPMCP/ Soluplus system correlates to limited agglomeration, high nilotinib release rate, and good storage stability.

Addressing the issue of surface stabilization of drug nanoparticles, although not prepared by $\mathrm{CO}_{2}$ precipitation, Van Eerdenbrugh et al. investigated 13 different surfactant and polymer stabilizers for 9 structurally different drugs (31). Generally, homopolymers, both synthetic and those of natural origin (e.g., alginate and poly(vinyl alcohol) (PVA)) were unable to stabilize the nanoparticles, although PVP displayed somewhat better performance. Also a number of cellulose derivatives, e.g., hydroxypropyl methylcellulose (HPMC), methyl cellulose (MC), hydroxyethyl cellulose (HEC), hydroxypropyl cellulose (HPC), and sodium carboxymethyl cellulose (NaCMC), displayed relatively modest stabilization of the drug nanoparticles, presumably due to a modest surface accumulation of these polymers at the drug particles at the low polymer concentrations used. In contrast, block copolymers (Poloxamer 188 and PVA-poly(ethylene glycol) graft copolymer) were more efficient in drug particle stabilizations, while the most effective particle stabilization was achieved by Polysorbate 80 and D, $\alpha$-tocopherol-poly(ethylene glycol)-1000succinate (TPGS), the latter able to stabilize about $80 \%$ of 
the drugs investigated. Furthermore, it was demonstrated that particle stabilization was directly correlated to the density of surfactant/polymer adsorbed to the particle surfaces. Also other studies have addressed these issues, observing stabilizing effects of, e.g., cellulose ethers such as HPMC and EHEC (32-34). Thus, nanoparticle stabilization through adsorption requires highly surface active polymers and surfactants, while stabilization through matrix formation places weaker requirements on this.

In a parallel study, Van Eerdenbrugh et al. investigated the stabilization of nanoparticles generated from 9 different drugs by TPGS. While the surfactant was able to provide protection against agglomeration, it was unable to halt Ostwald ripening, i.e., the growth of larger particles on expense of smaller ones through curvature-dependent solubility (35). Similarly, Lee et al. investigated a number sparingly soluble drug particles stabilized by either HPC, PVP, Pluronic F127, PEG, or Pluronic F68, and found that drugs with a lower solubility were more straightforwardly stabilized against particle growth (through Ostwald ripening) than more extensively soluble ones (36). Notably, surface active Pluronic (PEO-PPO-PEO) block copolymers, and to some extent also PVP and HPC, were able to stabilize particles formed by drugs of poor solubility, whereas the less surface active PEG was considerably less efficient.

As thus seen, numerous factors contribute to making polymer encapsulation of amorphous drug nanoparticles a complicated process. However, as long as the low drug solubility in aqueous solution is not primarily due to high stability of the solid state, and low enough to avoid Ostwald ripening (as with PKIs), and a suitable polymer for matrix formation (preferably with a high $\mathrm{T}_{\mathrm{g}}$ ) can be identified, hybrid nanoparticles are efficient in enhancing dissolution rate and improving oral bioavailability of sparingly soluble drugs. As demonstrated for PKIs in the present investigation, presence of surfactant is critical for maximally enhanced in vitro dissolution rates and GI absorption to be reached. In particular, it is important not only to consider the total amount of surfactant present in the nanoparticles, but also its distribution within the particles, as clearly demonstrated by the pronounced difference in PKI release rate for hybrid nanoparticles for which surfactant was dissolved together with polymer and drug in the organic solvent prior to $\mathrm{CO}_{2}$ precipitation (bulk distribution), and those for which surfactant was added after particle formation (surface distribution).

\section{CONCLUSIONS}

Hybrid nanoparticles, formed by $\mathrm{CO}_{2}$-induced precipitation of organic solutions containing a protein kinase inhibitor $(\mathrm{PKI})$ and a matrix-forming polymer, display dramatically improved in vitro dissolution kinetics and GI absorption of PKIs. In such hybrid materials, amorphous PKI is embedded within a polymer matrix, preventing PKI crystallization and particle growth. As demonstrated from increased dissolution rate, the approach taken allows considerable flexibility in polymer concentration, drug load, and nature of the matrix-forming polymer. Surfactants added after hybrid nanoparticle generation is important for optimal PKI dissolution rate, presumably a consequence of increased swelling and dissolution of the polymer matrix, as well as facilitated PKI solubilization during particle dissolution. With this, however, dramatically increased dissolution rate was observed for both simulated gastric fluid and simulated intestinal fluid. The increased dissolution rate observed in vitro translated into a strongly improved bioavailability in vivo for male beagle dogs following oral administration. Finally, the generality of the formulation approach taken was demonstrated for a range of PKIs.

\section{ACKNOWLEDGMENTS AND DISCLOSURES}

This work was supported by the Swedish Research Council (project 2012-1842) and XSpray Microparticles AB. Animal experiments were performed by Absorption Systems, Inc., USA.

\section{REFERENCES}

1. Di Gion P, Kanefendt F, Lindauer A, Doroshyenko O, Fuhr U, Wolf $\mathrm{J}$, et al. Clinical pharmacokinetics of tyrosin kinase inhibitors: focus on pyrimidines, pyridines, and pyrroles. Clin Pharmacokin. 2011;50:551-603.

2. Lowery A, Han ZZ. Assessment of tumor response to tyrosine kinase inhibitors. Front Biosci Landmark. 201 1;16:1996-2007.

3. Lindfors L, Forssen S, Westergren J, Olsson U. Nucleation and crystal growth in supersaturated solutions of a model drug. J Colloid Interface Sci. 2008;325:404-13.

4. Douroumis D, Fahr A. Stable carbamazepine colloidal systems using the cosolvent technique. Eur J Pharm Sci. 2007;30:367-74.

5. Puri V, Dantuluri AK, Bansal AK. Investigation of atypical dissolution behaviour of an encapsulated solid dispersion. J Pharm Sci. 2011;100:2460-8.

6. Pasquali I, Bettini R. Are pharmaceutics going supercritical? Int J Pharm. 2008;364:176-87.

7. Moribe K, Tozuka Y, Yamamoto K. Supercritical carbon dioxide processing of active pharmaceutical ingredients for polymorphic control and for complex formation. Adv Drug Delivery Rev. 2008;60:328-38.

8. Reverchon E, Adami R, Cardea S, Della Porta G. Supercritical fluids processing of polymers for pharmaceutical and medical applications. J Supercrit Fluids. 2009;47:484-92.

9. Byrappa K, Ohara S, Adschiri T. Nanoparticle synthesis using supercritical fluid technology - towards biomedical applications. Adv Drug Delivery Rev. 2008;60:299-327.

10. Martin A, Cocero MJ. Micronization processes with supercritical fluids: fundamentals and mechanisms. Adv Drug Delivery Rev. 2008;60:339-50. 
11. Pasquali I, Bettini R, Giordano F. Supercritical fluid technologies: an innovative approach for manipulating the solid state in pharmaceuticals. Adv Drug Delivery Rev. 2008;60:399-410.

12. Okamoto H, Danjo K. Application of supercritical fluid to preparation of powders of high-molecular weight drugs for inhalation. Adv Drug Delivery Rev. 2008;60:433-46.

13. Tom JW, Debenedetti PG. Particle formation with supercritical fluids - a review. J Aerosol Sci. 1991;22:555-84.

14. Cocero MJ, Martin A, Mattea F, Varona S. Encapsulation and coprecipitation processes with supercritical fluids: fundamentals and applications. J Supercrit Fluids. 2009;47:546-55.

15. Takishima S, O'Neill ML, Johnston KP. Solubility of block copolymer surfactants in compressed $\mathrm{CO}_{2}$ using a lattice fluid hydrogenbonding model. Ind Eng Chem Res. 1997;36:2821-33.

16. O’Neill ML, Cao Q Fang M, Johnston KP, Wilkinson SP, Smith CD, et al. Solubility of homopolymers and copolymers in carbon dioxide. Ind Eng Chem Res. 1998;37:3067-79.

17. Duarte ARC, Costa MS, Simplicio AL, Cardoso MM, Duarte CMM. Preparation of controlled release microspheres using supercritical fluid technology for delivery of anti-inflammatory drugs. Int J Pharm. 2006;308:168-74.

18. Breitenbach A, Mohr D, Kissel T. Biodegradable semi-crystalline comb polyesters influence the microsphere production by means of a supercritical extraction technique (ASES). J Control Release. 2000;63:53-60.

19. Bodmeier R, Wang H, Dixon DJ, Mawson S, Johnston KP. Polymeric microspheres prepared by spraying into compressed carbon dioxide. Pharm Res. 1995;12:1211-7.

20. Tian F, Sandler N, Aaltonen J, Lang C, Saville DJ, Gordon KC, et al . Influence of polymorphic form, morphology, and excipient interactions on the dissolution of carbamazepine compacts. J Pharm Sci. 2007;96:584-94.

21. Liu H, Finn N, Yates MZ. Encapsulation and sustained release of a model drug, indomethacin, using $\mathrm{CO}_{2}$-based microencapsulation. Langmuir. 2005;21:379-85.

22. Jantratid E, Dressman J. Biorelevant dissolution media simulating the proximal human gastrointestinal tract: an update. Dissolut Technol. 2009;8:21-5.
23. Couchman PR. Glass-transition temperatures of compatible polymer mixtures. Phys Lett. 1979;70A:155-7.

24. Kim D, Srivastava S, Narayanan S, Archer LA. Polymer nanocomposites: polymer and particle dynamics. Soft Matter. 2012;8:10813-8.

25. Malmsten M. Soft drug delivery systems. Soft Matter. 2006;2:760-9.

26. Malmsten M. Phase transformations in self-assembly systems for drug delivery applications. J Disp Sci Technol. 2007;28:63-72.

27. Lim RTY, Ng WK, Tan RBH. Amorphization of pharmaceutical compounds by co-precipitation using supercritical anti-solvent (SAS) process (Part I). J Supercrit Fluids. 2010;53:179-84.

28. Matsuda Y, Kawaguchi S, Kobayashi H, Nishijo H. Physicochemical characterization of spray-dried phenylbutasone polymorphs. J Pharm Sci. 1984;73:173-8.

29. Kluge J, Fusaro F, Muhrer G, Thakur R, Mazzotti M. Rational design of drug-polymer co-formulations by $\mathrm{CO}_{2}$ anti-solvent precipitation. J Supercrit Fluids. 2009;48:176-82.

30. Evans DE, Wennerström H. The colloidal domain - where physics, chemistry, biology, and technology meet. New York: Wiley-VHC; 1999.

31. Van Eerdenbrugh B, Vermant J, Martens JA, Froyen L, Van Humbeeck J, Augustijns P, et al. A screening study of surface stabilization during the production of drug nanocrystals. J Pharm Sci. 2009;98:2091-103.

32. Raseneck N, Hartenhauer H, Müller BW. Microcrystals for dissolution rate enhancement of poorly water-soluble drugs. Int J Pharm. 2003;254:137-45.

33. Malmsten M, Claesson PM. Temperature-dependent adsorption and surface forces in aqueous ethyl(hydroxyethyl)cellulose solutions. Langmuir. 1991;7:988-94.

34. Malmsten M, Tiberg F. Adsorption of ethyl(hydroxyethyl)cellulose at polystyrene. Langmuir. 1993;9:1098-103.

35. Van Eerdenbrugh B, Froyen L, Van Humbeeck J, Martens JA, Augustijns P, Van den Mooter G. Drying of crystalline drug nanosuspensions - the importance of surface hydrophobicity on dissolution behavior upon redispersion. Eur J Pharm Sci. 2008;35:127-35.

36. Lee J, Choi JY, Park CH. Characteristics of polymers enabling nanocomminution of water-insoluble drugs. Int J Pharm. 2008;355:328-36. 